\title{
Photoluminescence fine structures in the fractional quantum Hall effect regime
}

\author{
S. Nomura* \\ Division of Physics, University of Tsukuba, Tennodai, Tsukuba, 305-8571, Japan \\ M. Yamaguchi, H. Tamura, and T. Akazaki \\ NTT Basic Research Laboratories, NTT Corporation, Morinosato-Wakamiya, Atsugi, 243-0198, Japan \\ Y. Hirayama \\ Department of Physics, Faculty of Science, Tohoku University, Aoba, Aobaku, Sendai, 980-8578, Japan; \\ WPI-AIMR, Tohoku University, Katahira, Aobaku, Sendai, 980-0812, Japan; \\ and ERATO-JST, Katahira, Aobaku, Sendai, 980-0812, Japan \\ M. Korkusinski and P. Hawrylak ${ }^{\dagger}$ \\ Emerging Technologies Division, National Research Council, Ottawa, Ontario, Canada \\ (Received 19 July 2013; revised manuscript received 10 March 2014; published 25 March 2014)
}

\begin{abstract}
We investigate polarization-resolved fine structure in the photoluminescence (PL) in the fractional quantum Hall effect regime at $B=4-6 \mathrm{~T}$, where small Zeeman energy allows spin-depolarized ground states. We observe up to five distinct peaks with characteristic polarization and temperature dependence in the vicinity of $v=1 / 3$ and quenching of the PL from triplet charged quasiexcitons at around $v=1 / 4$. Those findings appear to be consistent with results of exact diagonalization on a Haldane sphere including all spin configurations and are understood to be PL from fractionally charged quasiexcitons.
\end{abstract}

DOI: 10.1103/PhysRevB.89.115317

PACS number(s): 71.35.Pq, 71.35.Ji, 73.43.Cd, 78.67.De

\section{INTRODUCTION}

The quest for the understanding of strongly correlated electrons in the fractional quantum Hall effect (FQHE) [1,2] continues $[3,4]$. At present, the FQHE is understood as the integer quantum Hall effect of composite fermions (CFs) [5,6]. A CF is composed of an electron with an integer number of flux quanta $\varphi_{0}=h / e$ attached to it. The effective magnetic field $B^{*}=B-2 q \nu B$ experienced by a $C F$ is reduced by $2 q v B$, where $2 q$ is the number of the attached flux quanta for $q=1,2, \ldots$, and $v$ is the electron filling factor. Hence, the $v=1 / 3$ state corresponds to the spin-polarized filled lowest Landau level of CFs with two flux quanta attached [7], while $v=1 / 4$ is expected to correspond to the Fermi sea of CFs with four flux quanta attached.

For FQHE at low magnetic fields the Zeeman energy is expected to be much smaller than the Coulomb energy, and the spin is expected to play an important role $[8,9]$. The spin polarization of the $\mathrm{CF}$ at low temperatures changes with $v$ due to the alignment of the CF Fermi level with respect to the up- and down-spin CF levels depending on $B^{*}$ [7,9-11]. The ground state of the two-dimensional electron system (2DES) at $v=1 / 3$ is expected to be fully polarized in the absence of the Zeeman energy. The spin configuration of the ground state of 2DES slightly away from $v=1 / 3$ was verified to involve a reversal of a large number of spins at small Zeeman energies $[9,12]$. The charged excitations in the vicinity of $v=1 / 3$ were thus shown to be skyrmions of $\mathrm{CFs}$, but it was claimed that extremely low density GaAs samples were required for the observation of skyrmions of CFs $[9,12]$.

\footnotetext{
*nomura.shintaro.ge@u.tsukuba.ac.jp

†Pawel.Hawrylak@nrc-cnrc.gc.ca
}

Upon an optical excitation, an electron-hole pair is created in the CF sea. The transition energies and the intensities of the photoluminescence (PL) due to recombination of the electron in the FQHE and a mobile hole have been found to correlate with $v$ [13-19], but only recently has a behavior characteristic of CFs been observed [18]. Optical methods have been used as important probes of the FQHE including the excited states and the spin polarization [10,20-22].

The early report on the observation of the $\sigma^{+}$-polarized PL in the FQHE regime suggests the possibility of extracting the spin polarization from the $\sigma^{+}$component of the PL. [16] However, the understanding of the PL in the FQHE regime, especially the PL in the $\sigma^{+}$polarization, has been limited until now despite extensive theoretical investigations [18,23-27]. For example, the effect of the electron-hole Coulomb interaction on the spin configuration of the electrons-hole system in the FQHE state has not been fully understood.

In this work, we address the role of spin in emission from FQHE by investigating the evolution of the circularly polarized $\mathrm{PL}$ in the FQHE regime at low perpendicular magnetic fields $B=4-6 \mathrm{~T}$ and as a function of the carrier density $n_{\mathrm{S}}$. A gated $\mathrm{GaAs} / \mathrm{Al}_{0.33} \mathrm{Ga}_{0.67} \mathrm{As}$ undoped quantum well (QW) sample was employed, in which the electron density can be tuned from nominally zero by bias voltage. The sample exhibits only small degradation of mobility in the low-density regime because of the lack of the donor layer [28-30]. We observe polarization-resolved fine structure in the vicinity of $v=1 / 3$ and a quenching of PL in the vicinity of $v=1 / 4$. The observed fine structure in circularly polarized PL is explained by calculated emission spectra for an increasing number of electrons interacting with an exciton $X$ on the Haldane sphere, including all spin configurations. The calculated results are consistent with our observations and relate the observed PL 
peaks to fractionally charged quasiexcitons in the interacting electron fluid.

\section{EXPERIMENT}

The PL experiments were carried out on a sample consisting of the $n$-GaAs substrate, a superlattice barrier, a 20-nm $\mathrm{Al}_{0.33} \mathrm{Ga}_{0.67} \mathrm{As}$ barrier layer, a 50-nm GaAs QW, and a 400-nm $\mathrm{Al}_{0.33} \mathrm{Ga}_{0.67} \mathrm{As}$ barrier layer. The electron density $n_{s}$ was tuned by applying a bias voltage $V_{B}$ to a bottom gate as given by $n_{s}=1.03 \times 10^{11} V_{B}+3.43 \times 10^{10}\left(\mathrm{~cm}^{-2}\right)$ for $V_{B}$ in $\mathrm{V}$. The sample had a peak mobility of $3 \times 10^{6} \mathrm{~cm}^{2} / \mathrm{Vs}$. The sample was mounted with silver paste on a copper plate that penetrates the die cavity of a chip carrier to a copper block thermally anchored to the mixing chamber. The sample was irradiated below the band gap of the barriers by linearly polarized laser light at $1.550 \mathrm{eV}$ from a Ti:sapphire laser that was introduced in a dilution refrigerator using a polarization-maintaining single-mode optical fiber at an incident power of $0.8 \mu \mathrm{W}$. The diameter of the laser spot was about $1 \mathrm{~mm}$. The excitation power density was kept small $\left(0.1 \mathrm{~mW} \mathrm{~cm}^{-2}\right)$ to minimize heating of the carriers.

The circular polarization-resolved PL measurements were performed at temperatures between 25 and $1000 \mathrm{mK}$ in perpendicular magnetic fields using a quarter-wave plate and a polarizer in the detection optical path. The PL was detected with a Spex 1404 double monochromator equipped with a liquid-nitrogen-cooled charge-coupled-device detector. Details of the experiments can be found elsewhere [31,32].

\section{PHOTOLUMINESCENCE FROM A GATED QUANTUM WELL}

Figure 1 shows the schematics of recombination processes between a conduction band (CB) electron in an $N_{L}=0$ Landau level and a valence band (VB) hole for $\sigma^{-}$and $\sigma^{+}$ polarizations. Figure 2 shows color plots representing the PL spectra from a gated QW at magnetic fields 4-6 T for $0.1<v<0.5$. To better visualize the observed features, the energy shift due to the vertical electric field has been subtracted from the photon energy. The energies of the lowest PL peak $\left(X_{\mathrm{s}}^{-}\right)$in $\sigma^{-}$polarization were fitted to a third-order polynomial function. The fitted functions were used as a "baseline" and were subtracted from the PL peak energies.

The lowest-energy transition is assigned to the spin-singlet charged exciton $X_{s}^{-}$, while the higher-energy peaks are (a)

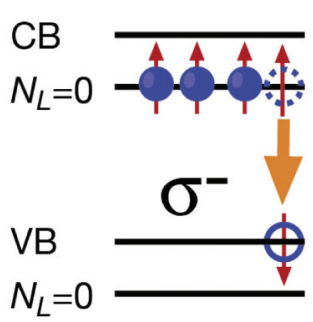

(b)

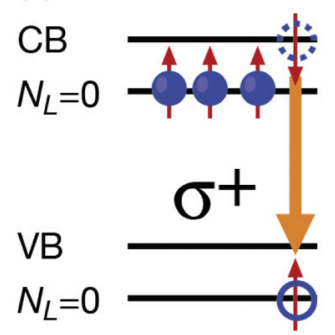

FIG. 1. (Color online) Schematics of recombination processes for (a) $\sigma^{-}$and (b) $\sigma^{+}$polarizations.

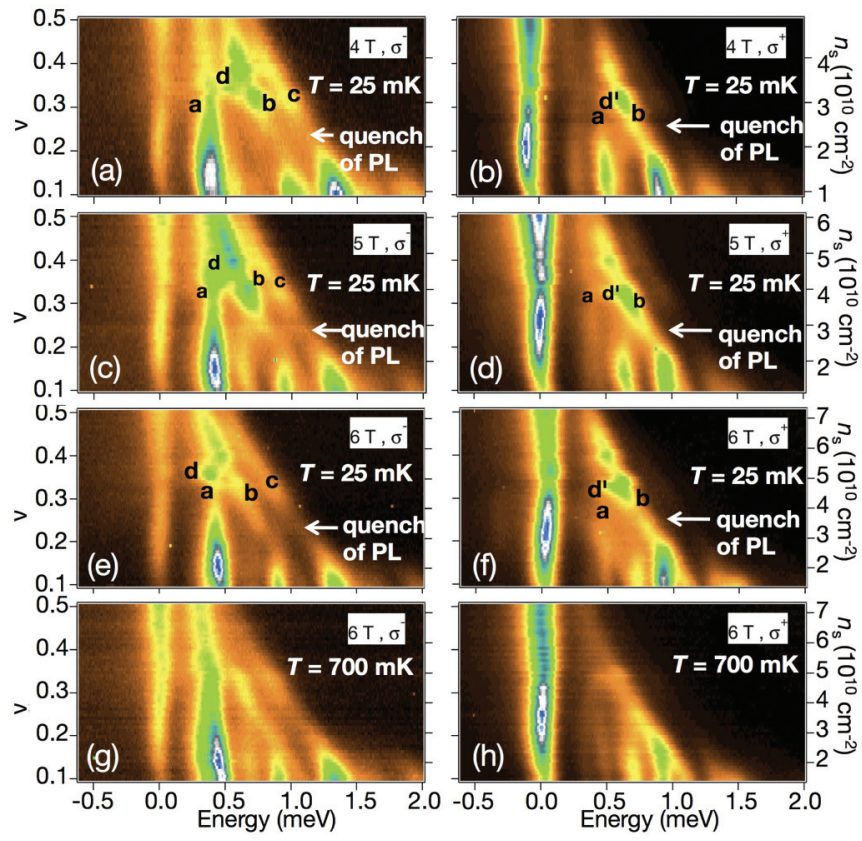

FIG. 2. (Color online) Color plots representing the PL spectra in a gated QW with a width of $50 \mathrm{~nm}$ in (a) $\sigma^{-}$and (b) $\sigma^{+}$polarizations at $4 \mathrm{~T}$, in (c) $\sigma^{-}$and (d) $\sigma^{+}$polarizations at $5 \mathrm{~T}$, in (e) $\sigma^{-}$and (f) $\sigma^{+}$ polarizations at $6 \mathrm{~T}$ at $25 \mathrm{mK}$, and in $(\mathrm{g}) \sigma^{-}$and $(\mathrm{h}) \sigma^{+}$polarizations at $6 \mathrm{~T}$ at $700 \mathrm{mK}$.

attributed to spin-triplet charged quasiexcitons immersed in the incompressible liquid [17,25]. Figures 2(a), 2(c), and 2(e) show the $v$-dependent majority-spin recombination spectrum with photon polarization $\sigma^{-}$at $25 \mathrm{mK}$ at 4,5 , and $6 \mathrm{~T}$, respectively. As $v$ increases, several striking features are visible. At around $v=1 / 4$, the PL intensities of the highest two to three peaks are quenched at 4,5 , and $6 \mathrm{~T}$, as shown in Figs. 2(a), 2(c), and 2(e), respectively. As $v$ increases, the emission recovers, but discontinuous jumps in energy and splittings at around $v=1 / 3$ are observed for the triplet charged quasiexciton PL emission lines, as shown in Figs. 2(a), 2(c), and $2(\mathrm{e})$.

The fine structures in PL are observed not only in $\sigma^{-}$ polarization but also in $\sigma^{+}$polarization [Figs. 2(b), 2(d), and 2(f)], where the emission is due to recombination of the minority-spin electron with the hole. At this polarization, the emission is dominated by $X_{s}^{-}$since $X_{s}^{-}$requires the minority spin. Still, we observe quenching of emission at around $v=1 / 4$.

The effect of temperature on PL is shown in Figs. 2(g) and 2(h). At $700 \mathrm{mK}$, most of the anomalies in the PL spectra disappear for both polarizations. Interestingly, the PL intensity at $v=1 / 4$ was recovered at $700 \mathrm{mK}$.

The PL spectra in the vicinity of $v=1 / 3$ are displayed in Fig. 3, and the PL peak energies in the vicinity of $v=1 / 3$ are displayed in Fig. 4 at 4-6 T. The area of circles denotes the peak intensity. Discontinuous jumps in energy and splittings of PL peaks at around $v=1 / 3$ are more clearly seen in both $\sigma^{-}$and $\sigma^{+}$polarizations. Remarkably, we find that PL peak d in Figs. 2(a), 2(c), and 2(e) is only observed at $v \geqslant 1 / 3$ and is absent at $v<1 / 3$. The PL spectrum at $4 \mathrm{~T}$ in Fig. 3(a) also 

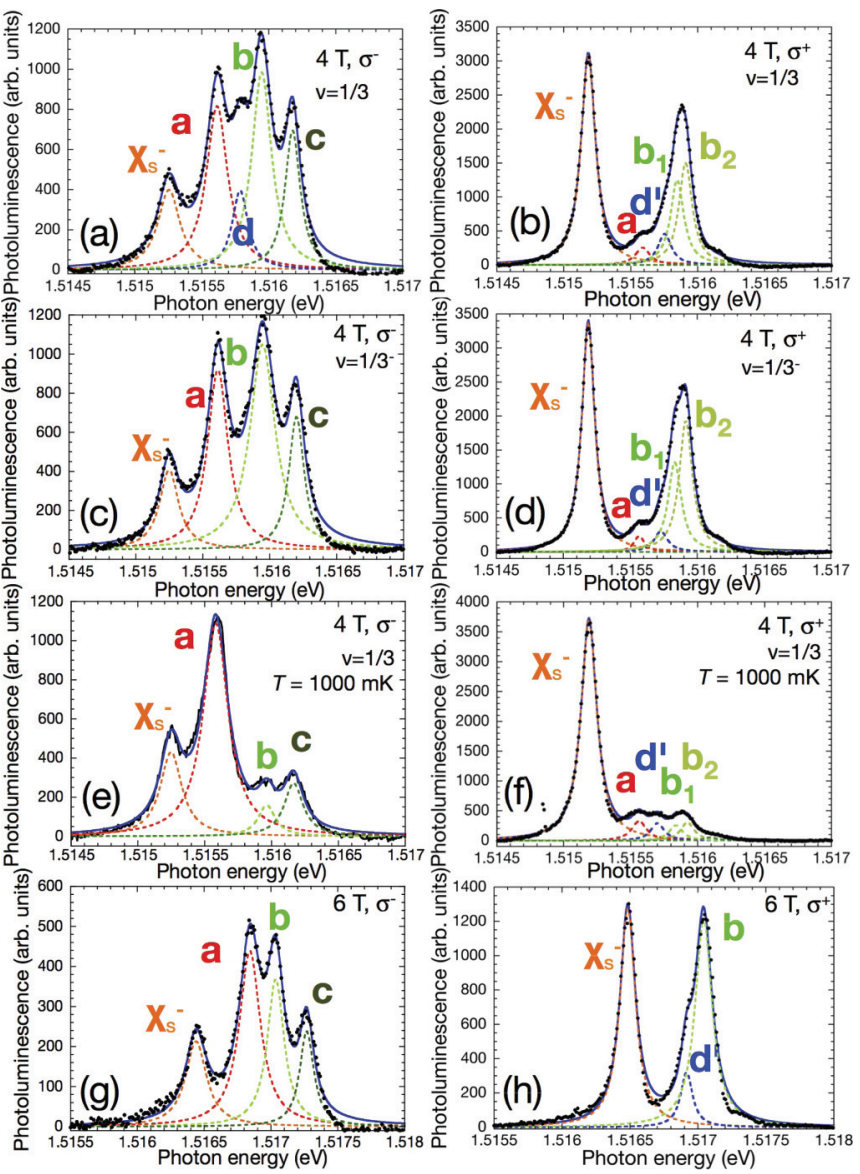

FIG. 3. (Color online) PL spectra at $v=1 / 3$ for (a) $\sigma^{-}$and (b) $\sigma^{+}$polarizations at $T=25 \mathrm{mK}$ at $4 \mathrm{~T}$. PL spectra at $v=1 / 3^{-}(0.323)$ for (c) $\sigma^{-}$and (d) $\sigma^{+}$polarizations at $T=25 \mathrm{mK}$. PL spectra at $v=1 / 3$ for (e) $\sigma^{-}$and (f) $\sigma^{+}$polarizations at $T=1000 \mathrm{mK}$. PL spectra at $v=1 / 3$ for $(\mathrm{g}) \sigma^{-}$and (h) $\sigma^{+}$polarizations at $T=25 \mathrm{mK}$ at $6 \mathrm{~T}$. Fitted curves are also shown.

clearly shows PL peak $\mathrm{d}$ at $v=1 / 3$ and the absence of PL peak d at $v=1 / 3^{-}$in Fig. 3(c). Peak d also disappears at $T \geqslant$ $700 \mathrm{mK}$, as shown in Figs. 2(g) and 3(e). Peak d is observed only due to the presence of quasielectrons. Furthermore, we can identify a transition marked by d' that is observed in the presence of quasiholes at $v \leqslant 1 / 3$ as a shoulder, as shown in Figs. 2(b), 2(d), and 2(f) and 3(b), 3(d), 3(f), and $3(\mathrm{~h})$.

The lowest peak in Figs. 2-4 is assigned to be $X_{s}^{-}$. This state is the most strongly bound state in the presence of quasielectrons or quasiholes. No discontinuities of the transition energy of $X_{s}^{-}$were observed at $v=1 / 3$. The PL peak intensity for $\sigma^{+}$is larger than for $\sigma^{-}$polarization. This is due to large excitonic enhancement of the oscillator strength induced by rearranging the occupations of the spindown electron in response to the optically created hole [32]. This effect is more significant at lower magnetic fields, as observed in Figs. 4(e)-4(g). The observation of fine structures in PL may be due to the lack of a donor layer and hence the lack of short-distance potential fluctuation originating from the remote ionized donor layer in modulation-doped GaAs QWs.

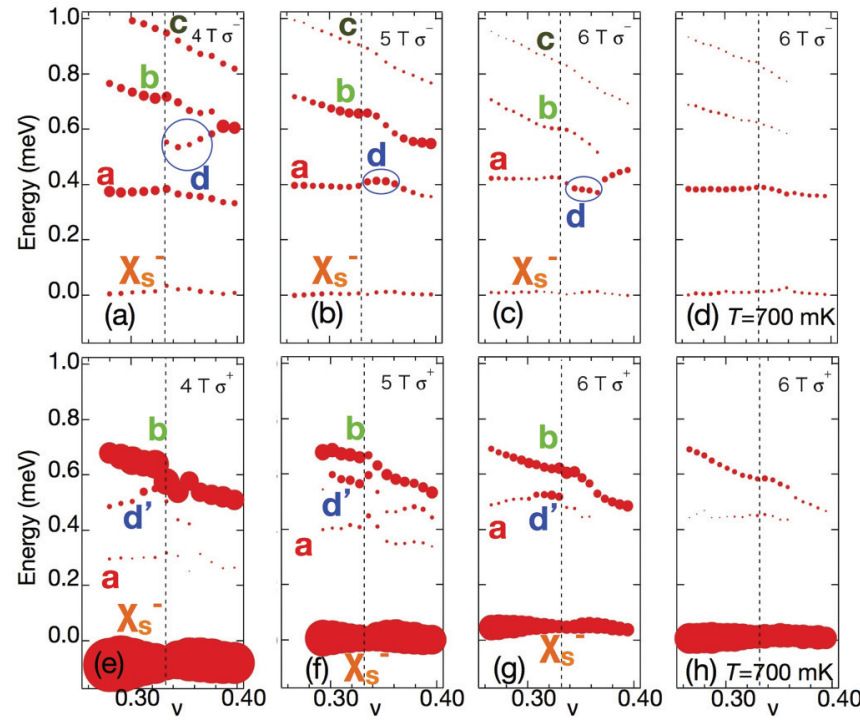

FIG. 4. (Color online) PL peak energies at (a) $4 \mathrm{~T}$, (b) $5 \mathrm{~T}$, and (c) $6 \mathrm{~T}$ at $25 \mathrm{mK}$ and (d) $6 \mathrm{~T}$ at $700 \mathrm{mK}$ for $\sigma^{-}$polarization and PL peak energies at (e) $4 \mathrm{~T}$, (f) $5 \mathrm{~T}$, and (g) $6 \mathrm{~T}$ at $25 \mathrm{mK}$ and (h) $6 \mathrm{~T}$ at $700 \mathrm{mK}$ for $\sigma^{+}$polarization as a function of $\nu$. The area of circles denotes PL peak intensity.

\section{EMISSION SPECTRA FOR ELECTRONS INTERACTING WITH AN EXCITON ON THE HALDANE SPHERE}

To understand the above observations, following earlier work $[18,23,26,33,34]$, we study recombination spectra of an exciton interacting with an increasing number of electrons $N_{e}$ on the Haldane sphere [35]. An infinite two-dimensional plane is mapped onto the Haldane sphere, at the center of which there is a magnetic monopole of strength $2 S$. The degeneracy of the lowest Landau level $g=2 S+1$, and $v$ is determined by $v=\left(N_{e}-1\right) /(2 S)$, where $S^{*}=2 S$ is proportional to the number of flux quanta. We compute the initial ground and excited states of the photoexcited state of $N_{e}+1$ electrons and the single valence hole including all spin configurations. Upon recombination, the electronic system is left in any of its allowed final $N_{e}$ electron states. All calculations are performed for subspaces characterized by the total spin projection of the electrons $S_{e}^{z}$, and the total spin is extracted from electronic degeneracies. Zeeman energy equal to zero was assumed. Calculations are applicable to triplet charged quasiexciton states since $X_{s}^{-}$requires the inclusion of the Landau-level mixing.

In the following we focus on the vicinity of $v=1 / 3$. The energy levels as a function of total angular momentum $L$ of the photoexcited system consisting of $N_{e}=5$ electrons plus one $X$ at $v=1 / 3\left(S^{*}=12\right)$ are shown in Figs. 5(a) and 5(b) for the spin-polarized and spin-depolarized systems, respectively. The energy is measured from the ground-state energy in units of the gap $E_{\mathrm{g}}$ at $v=1 / 3$. The low-energy spectra for the spin-polarized $S_{e}=3$ system [Fig. 5(a)] correspond to a charged exciton propagating in the incompressible fluid. The optically active initial states are indicated by triangles, whose area is proportional to the oscillator strength. The dominant emission for the spin-polarized system at a low temperature 

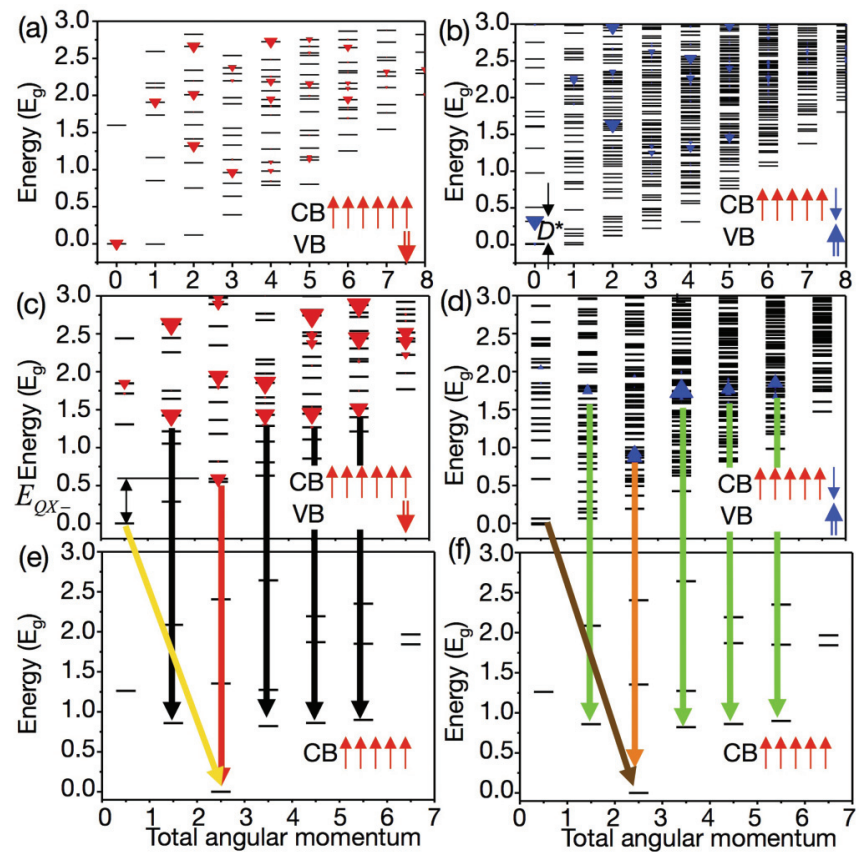

FIG. 5. (Color online) Energy levels as a function of total angular momentum $L$ of the photoexcited system consisting of $N_{e}=5$ electrons plus one $X$ for (a) $S_{e}=3$ and (b) $S_{e}=2$ at $v=1 / 3$ $\left(S^{*}=12\right)$. The area of the triangles reflects the oscillator strength for the transition to $N_{e}=5$ electrons, $S_{e}=5 / 2$ final state. Energy levels for (c) $S_{e}=3$ and (d) $S_{e}=2$ for $v=1 / 3^{+}\left(S^{*}=11\right)$. (e) and (f) Energy levels of the final state $N_{e}=5$ electrons for $S_{e}=5 / 2$ at $v=1 / 3^{+}\left(S^{*}=11\right)$ and spin projection $S_{e}=5 / 2$. The energy is measured from $E_{0}$. The arrows indicate optical transitions.

is the emission from the initial ground state with $S_{e}=3$ to the $S_{e}=5 / 2$ final state at $L=0$, corresponding to $\sigma^{-}$ polarization, as shown in Fig. 5(a). For a spin-depolarized, $S_{e}=2$, system in Fig. 5(b), the optically active state at $L=0$ is the second lowest state with energy $D^{*}$ above the ground-state energy. This state is populated at a finite temperature and gives the dominant emission in $\sigma^{+}$polarization.

We now turn to the emission spectrum in the presence of quasielectrons, $v>1 / 3$. Figures $5(\mathrm{c})-5(\mathrm{f})$ show the energy spectra of initial and final states as a function of $L$ at $v=1 / 3$ minus one flux quantum $\left(S^{*}=11\right)$. The initial spectrum of $N_{e}=5$ electrons plus one $X\left(N_{e}=6+1 h\right)$ with maximum total spin $S_{e}=3$ is shown in Fig. 5(c), while the system with a lower total spin, $S_{e}=2$, and with reversed spin excitations is shown in Fig. 5(d). Figures 5(e) and 5(f) show the finalstate spectrum of $N_{e}=5$ electrons with $S_{e}=5 / 2$. Schematic diagrams that outline the steps of optical recombination are shown in Figs. 6(a) and 6(b).

The $L=5 / 2$ ground state in the $N_{e}=5$ electron system is understood as a filled lowest CF Landau level and a single CF quasielectron in the second CF Landau level. The quasielectron binds to the quasiexciton, forming a fractionally charged quasiexciton. The dominant emission at a finite temperature (corresponding to peak b in Figs. 2 and 3) is the transition from the initial excited state at $L=5 / 2$ with $S_{e}=3$ or $S_{e}=2$ to the $S_{e}=5 / 2$ final state at $L=5 / 2$. These transitions are marked by red and orange arrows in Fig. 5. Interestingly, we have (a)
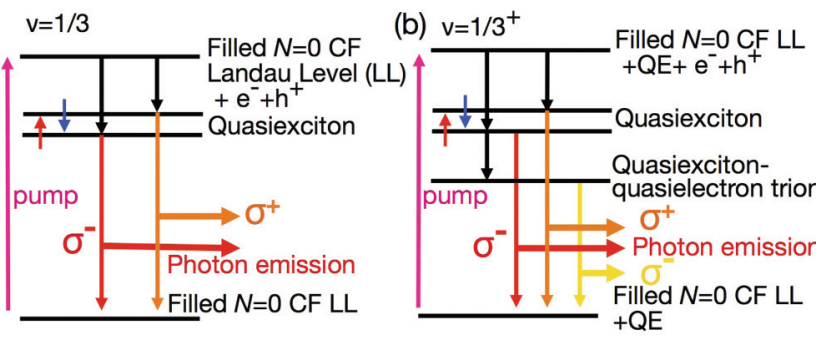

FIG. 6. (Color online) Schematic diagrams that outline the steps of optical recombination at (a) $v=1 / 3$ and (b) $v=1 / 3^{+}$.

found that the initial ground state at $L=1 / 2$ with $S_{e}=3$ and $S_{e}=2$ is optically inactive. The emission from the ground state is only possible with the help of impurity scattering at low temperature. The angular momentum nonconserving transition marked by the yellow arrow is also only allowed by impurity scattering. This transition leaves a single $\mathrm{CF}$ quasielectron in the second CF Landau level. In the case of recombination of the majority-spin electron with the hole, the recombination probability after impurity scattering is large because the spin selection rule is fulfilled for all majority-spin electrons. By contrast, the recombination probability is small for the case of recombination of the minority-spin electron after impurity scattering. This explains the observation of peak $\mathrm{d}$ only in $\sigma^{-}$polarization, as shown in Figs. 2 and 3 with the separation of peaks $\mathrm{d}$ and $\mathrm{b}\left(E_{\mathrm{bd}}\right)$ as the fractionally charged quasiexciton binding energy $E_{Q X}$. The gap energy $E_{\mathrm{g}}$ at $v=1 / 3$ is estimated to be $0.34 \mathrm{meV}$ at $6 \mathrm{~T}$ by taking $E_{Q X-}=E_{\mathrm{bd}}=0.20 \mathrm{meV}$. This result is consistent with the gap energy $E_{\mathrm{g}}=3 \mathrm{~K}=0.26 \mathrm{meV}$ estimated by a transport measurement of the activation gap at $6 \mathrm{~T}$ [12].

Figures 7(a) and 7(b) show calculated emission spectra at $v=1 / 3\left(S^{*}=12\right)$ and at $1 / 3^{+}\left(S^{*}=11\right)$, respectively, for recombinations of majority-spin and minority-spin electrons.
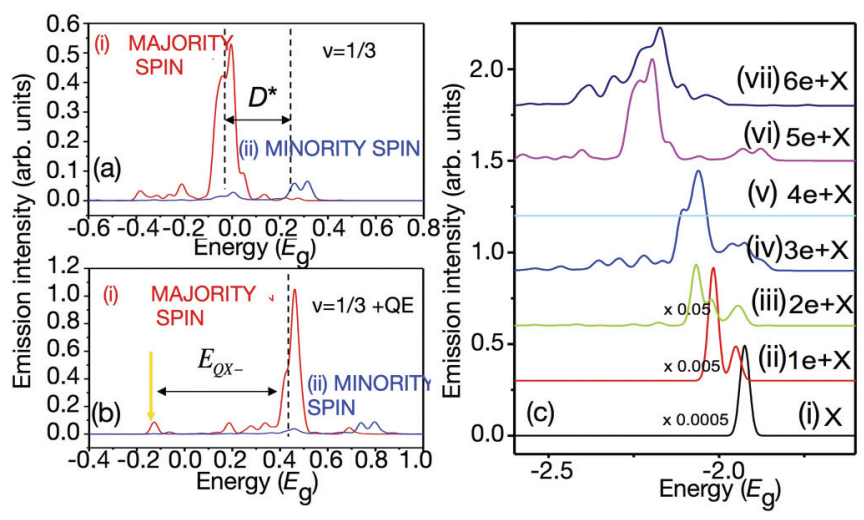

FIG. 7. (Color online) (a) Calculated emission spectra for recombination of (i) a majority-spin electron and (ii) a minority-spin electron at $v=1 / 3\left(S^{*}=12\right)$. (b) Same as in (a), but at $v=1 / 3^{+}$ $\left(S^{*}=11\right)$. The emission spectra are aligned to $v=1 / 3$, as indicated by the dashed line. The yellow line corresponds to the transition indicated by the yellow arrow in Fig. 5(e). (c) Evolution of emission spectra at fixed $B\left(S^{*}=12\right)$ for the initial states of (i) zero electrons (e), (ii) $1 e$, (iii) $2 e$, (iv) $3 e$, (v) $4 e$, (vi) $5 e$, and (vii) $6 e$ plus $X$ at temperature $T=0.4 E_{\mathrm{g}}$. Spectra are shifted vertically for clarity. 
The observed PL peaks in Figs. 2 and 3 are reproduced in Figs. 7(a) and 7(b). The emission spectra were calculated for all spin configurations, and we found no visible additional structures to Figs. 7(a) and 7(b). The peak with the largest oscillator strength in Figs. 7(a) and 7(b) for the majority- and minority-spin electrons corresponds to the largest PL peak, peak b in Figs. 2 and 3. The higher-energy peak in Figs. 7(a) and 7(b) for the recombination of the majority-spin electron corresponds to peak c in Figs. 2 and 3. The calculated emission spectrum lacks the corresponding higher-energy peak for the recombination of the minority-spin electron, in agreement with the observation in Figs. 2(b), 2(d), 2(f), 3(b), 3(d), 3(f), and $3(\mathrm{~h})$. The peak with the largest oscillator strength for $\sigma^{+}$is higher in energy than the corresponding peak for $\sigma^{-}$ polarization by $D^{*}$, while the peak energy of peak b for $\sigma^{+}$in Fig. 2 is lower than that of peak b for $\sigma^{-}$. This is because the Landau-level mixing is not taken into account in the calculations. The reduction of the peak energies and the enhancement of the PL for $\sigma^{+}$in Fig. 2 is due to the spin-dependent excitonic effect [32]. The transition marked by the yellow arrow in Fig. 7(b) is due to the recombination of the fractionally charged quasiexciton state corresponding to peak $\mathrm{d}$ in $\sigma^{-}$polarization in Figs. 2 and 3. The lower-energy PL peak (peak a) that increases with temperature may be due to disorder-induced emission from a nonmultiplicative dark triplet trion state not shown in Figs. 7(a) and 7(b).

The evolution of calculated emission spectra at a fixed $B$ $\left(S^{*}=12\right)$ for the initial states of $N_{e}$ electrons plus $X$ at $T=$ $0.4 E_{\mathrm{g}}$ is shown in Fig. 7(c). We see how the multiple peaks at $N_{e}=5$ and 6 are developed from the single peak at $N_{e}=0$ and the double peak at $N_{e}=1$. A remarkable result is the quenching of the oscillator strength at $v=1 / 4$ for $N_{e}=4$ electrons plus $X$. This was also obtained at $v=1 / 4$ for five electrons plus $X$ at $S^{*}=16$. We have found that the low-lying states in all spin subspaces are dark at $v=1 / 4$. This result agrees with the quenching of the PL of the highest two to three peaks at around $v=1 / 4$ in Figs. 2(a)-2(f). The recovery of the quenching of the PL at $700 \mathrm{mK}$ in Figs. 2(g) and 2(h) also confirms that the low-lying states in all spin subspaces are dark. The case of $v=1 / 4$ is expected to be a Fermi sea of CFs with four flux quanta attached, but the Wigner solid might be favored by disorder [15].

\section{CONCLUSIONS}

We have observed a distinct fine structure and discontinuous jumps in energy and splittings in PL at around $v=1 / 3$ and quenching of PL intensities of higher-energy peaks at around $v=1 / 4$. These features in PL are in accordance with calculated emission spectra of electrons interacting with an exciton on the Haldane sphere, including all spin configurations. We have observed a PL peak that shows large intensity only in the presence of quasielectrons, and it has been shown that this peak is due to recombination of a quasiexciton-quasielectron trion leaving the quasielectron in the excited state. Quenching of PL intensities of higher-energy peaks at around $v=1 / 4$ has been observed, in agreement with numerical calculations, which show the low-lying states in all spin subspaces to be dark at $v=1 / 4$.

\section{ACKNOWLEDGMENTS}

The authors are grateful to J. K. Jain for valuable discussions. This work was partly supported by Kakenhi No. 20104005 and No. 25103704.

\section{APPENDIX: BRIEF ACCOUNT OF THE THEORETICAL APPROACH}

We consider theoretically the system of $N_{e}$ interacting electrons accompanied by an exciton and confined in a quantum well. The quantum-well boundary conditions (translational invariance) are approximated by mapping the system onto the Haldane sphere with a magnetic monopole placed in its center. With a strength of the monopole of $2 S$, the lowest Landau level is composed of $g=2 S+1$ degenerate levels, labeled by the single-particle angular momenta $l=-S, \ldots, S$. A similar electronic structure is also assumed for the valence hole.

We populate the single-particle levels with $N_{e}+1$ electrons and one hole. The Hamiltonian of such a system of confined interacting carriers is

$$
\begin{aligned}
\hat{H}= & \frac{1}{2} \sum_{i j k l}^{2 S+1} \sum_{\sigma \sigma^{\prime}}\left\langle i \sigma j \sigma^{\prime}\left|V_{e e}\right| k \sigma^{\prime} l \sigma\right\rangle c_{i \sigma}^{+} c_{j \sigma^{\prime}}^{+} c_{k \sigma^{\prime}} c_{l \sigma} \\
& -\sum_{i j k l}^{2 S+1} \sum_{\sigma}\left\langle i \sigma j \tau\left|V_{e h}\right| k \tau l \sigma\right\rangle c_{i \sigma}^{+} h_{j \tau}^{+} h_{k \tau} c_{l \sigma},
\end{aligned}
$$

where $c_{i \sigma}^{+}\left(c_{i \sigma}\right)$ creates (annihilates) an electron in state $i$ with spin $\sigma= \pm 1 / 2$ and $h_{i \tau}^{+}\left(h_{i \tau}\right)$ creates (annihilates) a hole in state $i$ with spin $\tau= \pm 3 / 2$. The above Hamiltonian contains only the electron-electron and electron-hole Coulomb terms, whose characteristic matrix elements are analytical functions of the state indices and $2 S$.

First, we choose the projection of the total electronic spin $S_{e}$ by selecting the number of electrons with spin up and with spin down, as well as the spin $\tau$ of the hole. Further, we create all possible configurations of $N_{e}+1$ electrons and one hole on the single-particle levels and divide them into classes labeled by the total angular momentum $L=\sum_{i} l_{i}^{e}+l^{h}$. Within each $\left\{S_{e, z}, L\right\}$ subspace we construct the matrix of the Hamiltonian in the basis of the many-particle configurations and diagonalize this Hamiltonian numerically using a block, Jacobi-Davidsonlike conjugate gradient algorithm. A similar computational procedure is carried out for the system of $N_{e}$ electrons, that is, the initial system with one electron-hole pair removed. Last, we utilize Fermi's golden rule to compute the emission spectra from the system $N_{e}+X$ to the system with $N_{e}$ electrons. The procedure is described in detail in Ref. [36].
[1] D. C. Tsui, H. L. Stormer, and A. C. Gossard, Phys. Rev. Lett. 48, 1559 (1982).
[2] R. B. Laughlin, Phys. Rev. Lett. 50, 1395 (1983). 
[3] B. Yang, Z.-X. Hu, Z. Papić, and F. D. M. Haldane, Phys. Rev. Lett. 108, 256807 (2012).

[4] F. D. M. Haldane, Phys. Rev. Lett. 107, 116801 (2011).

[5] J. K. Jain, Phys. Rev. Lett. 63, 199 (1989).

[6] B. I. Halperin, P. A. Lee, and N. Read, Phys. Rev. B 47, 7312 (1993).

[7] K. Park and J. K. Jain, Phys. Rev. Lett. 80, 4237 (1998).

[8] T. Chakraborty, P. Pietiläinen, and F. C. Zhang, Phys. Rev. Lett. 57, 130 (1986).

[9] R. Kamilla, X. Wu, and J. Jain, Solid State Commun. 99, 289 (1996).

[10] I. V. Kukushkin, K. v. Klitzing, and K. Eberl, Phys. Rev. Lett. 82, 3665 (1999).

[11] M. Stern, P. Plochocka, V. Umansky, D. K. Maude, M. Potemski, and I. Bar-Joseph, Phys. Rev. Lett. 105, 096801 (2010).

[12] A. F. Dethlefsen, R. J. Haug, K. Výborný, O. Čertík, and A. Wójs, Phys. Rev. B 74, 195324 (2006).

[13] B. B. Goldberg, D. Heiman, A. Pinczuk, L. Pfeiffer, and K. West, Phys. Rev. Lett. 65, 641 (1990).

[14] A. J. Turberfield, S. R. Haynes, P. A. Wright, R. A. Ford, R. G. Clark, J. F. Ryan, J. J. Harris, and C. T. Foxon, Phys. Rev. Lett. 65, 637 (1990).

[15] H. Buhmann, W. Joss, K. von Klitzing, I. V. Kukushkin, G. Martinez, A. S. Plaut, K. Ploog, and V. B. Timofeev, Phys. Rev. Lett. 65, 1056 (1990).

[16] B. B. Goldberg, D. Heiman, M. Dahl, A. Pinczuk, L. Pfeiffer, and K. West, Phys. Rev. B 44, 4006 (1991).

[17] G. Yusa, H. Shtrikman, and I. Bar-Joseph, Phys. Rev. Lett. 87, 216402 (2001).

[18] M. Byszewski, B. Chwalisz, D. Maude, M. Sadowski, M. Potemski, T. Saku, Y. Hirayama, S. Studenikin, D. Austing, A. Sachrajda, and P. Hawrylak, Nat. Phys. 2, 239 (2006).
[19] S. Nomura, M. Yamaguchi, T. Akazaki, H. Tamura, H. Takayanagi, and Y. Hirayama, Phys. E 34, 292 (2006).

[20] M. Kang, A. Pinczuk, B. S. Dennis, M. A. Eriksson, L. N. Pfeiffer, and K. W. West, Phys. Rev. Lett. 84, 546 (2000).

[21] J. G. Groshaus, P. Plochocka-Polack, M. Rappaport, V. Umansky, I. Bar-Joseph, B. S. Dennis, L. N. Pfeiffer, K. W. West, Y. Gallais, and A. Pinczuk, Phys. Rev. Lett. 98, 156803 (2007).

[22] J. G. Groshaus, I. Dujovne, Y. Gallais, C. F. Hirjibehedin, A. Pinczuk, Y.-W. Tan, H. Stormer, B. S. Dennis, L. N. Pfeiffer, and K. W. West, Phys. Rev. Lett. 100, 046804 (2008).

[23] V. M. Apalkov and E. I. Rashba, Phys. Rev. B 46, 1628 (1992).

[24] X. M. Chen and J. J. Quinn, Phys. Rev. Lett. 70, 2130 (1993).

[25] A. Wojs and P. Hawrylak, Phys. Rev. B 51, 10880 (1995).

[26] A. Wójs and J. J. Quinn, Phys. Rev. B 63, 045304 (2000).

[27] A. Wójs, A. Gladysiewicz, and J. J. Quinn, Phys. Rev. B. 73, 235338 (2006).

[28] Y. Hirayama, K. Muraki, and T. Saku, Appl. Phys. Lett. 72, 1745 (1998).

[29] S. Nomura, M. Yamaguchi, T. Akazaki, H. Tamura, T. Maruyama, S. Miyashita, and Y. Hirayama, Phys. Rev. B 76, 201306(R) (2007).

[30] M. Yamaguchi, S. Nomura, T. Maruyama, S. Miyashita, Y. Hirayama, H. Tamura, and T. Akazaki, Phys. Rev. Lett. 101, 207401 (2008).

[31] M. Yamaguchi, S. Nomura, H. Tamura, and T. Akazaki, Phys. Rev. B 87, 081310 (2013).

[32] S. Nomura, H. Tamura, M. Yamaguchi, T. Akazaki, and Y. Hirayama, Phys. Rev. B 87, 085318 (2013).

[33] A. H. MacDonald and E. H. Rezayi, Phys. Rev. B 42, 3224 (1990).

[34] A. Wójs, J. J. Quinn, and P. Hawrylak, Phys. Rev. B. 62, 4630 (2000).

[35] F. D. M. Haldane, Phys. Rev. Lett. 51, 605 (1983).

[36] M. Korkusinski and P. Hawrylak (unpublished). 